\title{
Tuberculosis and Human Immunodeficiency Virus Co-Infection and Associated Factors at Debre Markos Comprehensive Specialized Hospital, Northwest Ethiopia: A Four-Year Retrospective Study
}

This article was published in the following Dove Press journal: HIVIAIDS - Research and Palliative Care

\author{
Adugnaw Alemu (D) \\ Moges Wubie Aycheh ${ }^{2}$ \\ Tebelay Dilnessa (1D ${ }^{3}$ \\ 'School of Medicine, Debre Markos \\ University, Debre Markos, Ethiopia; \\ ${ }^{2}$ Department of Public Health, College of \\ Health Sciences, Debre Markos \\ University, Debre Markos, Ethiopia; \\ ${ }^{3}$ Department of Medical Laboratory \\ Sciences, College of Health Sciences, \\ Debre Markos University, Debre Markos, \\ Ethiopia
}

Background: Human immunodeficiency virus (HIV) and tuberculosis (TB) are the principal global causes of death among patients with communicable diseases. Because of shared immune defense mechanisms, they are the primary cause of morbidity worldwide. However, little information was found regarding the magnitude of TB/HIV co-infection in the study area, in Northwest Ethiopia.

Objective: The main aim of this study was to assess the prevalence of TB and HIV coinfection and associated factors among TB patients.

Methods: All TB patients at Debre Markos Comprehensive Specialized Hospital were included from September 11, 2012 to September 10, 2016. Data were analyzed using SPSS version 22. Logistic regression was used to determine the associations between independent and outcome variables.

Results: A total of 180 TB patients were enrolled. Pulmonary tuberculosis (PTB) accounted for $97 / 180$ (53.9\%), followed by extrapulmonary tuberculosis (EPTB) in 83/180 (46.1\%). There were 164/180 (91.1\%) new TB cases and 16/180 (8.9\%) treatment failures, but no relapsing or defaulting cases were observed. Forty percent $(72 / 180)$ of patients were coinfected with TB and HIV. The likelihoods of having TB/HIV co-infection were 3.2 and 2.1 times higher in PTB smear-positive and PTB smear-negative patients (AOR=3.2, 95\% CI 1.4-8.1, $p=0.006$; and $\mathrm{AOR}=2.1,95 \%$ CI 1.0-4.3, respectively, $p=0.05$ ), in comparison to EPTB. The rate of TB/HIV co-infection was $28 / 66$ (42.4\%) in 2013, 18/38 (47.4\%) in 2014, $13 / 32(40.6 \%)$ in 2015 , and $13 / 44(29.5 \%)$ in 2016 .

Conclusion: TB/HIV co-infection showed a decreasing trend in the past 2 years in the study area. TB/HIV co-infection is one of the most serious community health concerns in the study area. Therefore, TB/HIV collaborative activities should be implemented to reduce coinfection and its impact on the community.

Keywords: TB/HIV co-infection, tuberculosis, HIV, multidrug resistant TB

\section{Background}

Tuberculosis (TB) is a chronic infectious disease that has been a major health problem over the centuries and has accounted for more human suffering, loss of earnings, and failure of economic and social development than any other disease. ${ }^{1}$
Correspondence: Tebelay Dilnessa Department of Medical Laboratory Sciences, College of Health Sciences, Debre Markos University, PO Box 269, Debre Markos, Ethiopia

$\mathrm{Tel}+25$ I 9121987/5

Email tebelay@gmail.com 
According to the WHO (2005), about one-third of the world's population is infected with Mycobacterium tuberculosis, about 9.6 million cases of active disease are estimated to occur each year, and annually 1.5 million people die of TB. ${ }^{2}$

HIV/AIDS has substantially altered the epidemiology of TB by increasing the risk of reactivating latent TB, increasing the chance of TB infection once exposed to tubercle bacilli (re-infection) and increasing the risk of rapid progression soon after infection. ${ }^{3,4}$ Among a globally estimated 9.6 million incident cases of TB in 2014, around $12 \%$ were co-infected with HIV. Also, from a total of 1.5 million TB deaths, 1.1 million and 0.4 million were HIV-negative and HIV-positive, respectively. ${ }^{5}$ The African region accounted for $74 \%$ of these cases. ${ }^{6}$ According to a WHO report, Ethiopia ranked seventh among 22 high-burden countries with TB and HIV infection in the world. ${ }^{1}$

TB and HIV co-infections are associated with special diagnostic and therapeutic challenges and constitute a huge burden on the healthcare systems of developing countries such as Ethiopia. ${ }^{7}$ A person infected with $M$. tuberculosis has a $10 \%$ risk of developing TB disease during their lifetime, but for people co-infected with both M. tuberculosis and HIV, the annual risk of developing active TB disease exceeds $10 \% .^{8}$ The complication of treatment outcomes of both diseases due to the TB/HIV co-infection results particularly from the concomitant use of anti-retroviral treatment (ART) and the intensive phase of TB treatment; in addition, it leads to drug-drug interactions, side effects of multiple drugs, increased pill burden, decreased adherence, and the development of higher rates of drug-resistant organisms because of the combination of these factors. ${ }^{9}$

Ethiopia is one of the developing countries with a high burden of TB/HIV co-infections. An Ethiopia National TB/HIV Sentinel Surveillance report showed that the prevalence of HIV among TB patients was $20 \%{ }^{10}$ Although both TB and HIV infections are preventable, the global burden of TB has increased since the epidemic of HIV/AIDS as it increases both new TB infections and latent TB, causing active TB as a result of compromised immunity in HIV/AIDS patients. ${ }^{11}$ The mortality and morbidity of patients with this co-infection is also high compared to isolated infections with TB or HIV. ${ }^{12}$ But there are insufficient data to show the burden of TB/HIV co-infection in developing countries such as Ethiopia, particularly in the study area. Therefore, the main aim of this study was to determine the prevalence of $\mathrm{TB}$ and HIV co-infection, and associated risk factors, among TB patients at the directly observed treatment, short-course (DOTS) clinic of Debre Markos Comprehensive Specialized Hospital, Ethiopia.

\section{Patients and Methods Study Setting and Context}

A retrospective cross-sectional study was conducted among TB patients at Debre Markos Comprehensive Specialized Hospital, Ethiopia, registered from September 11, 2012 to September 10, 2016 at the DOTS clinic. The hospital is a tertiary level center which provides health services to a population of over 3.5 million people. $^{13}$ The hospital provides TB and HIV/AIDS prevention and treatment services. The data were collected from March 14 to 21, 2017.

\section{Sample Size and Sampling Technique}

The sample comprised 180 patients registered at the DOTS clinic, with complete data in the log book of the DOTS clinic from September 11, 2012 to September 10, 2016. The sample included all TB patients with complete data during the study period. Participants in the Ethiopian National TB/HIV Sentinel Surveillance program of July 2011 to June 2012 were excluded from this study because the profiles of these patients had already been analyzed in that surveillance program.

\section{Inclusion Criteria}

All TB patients with records in the DOTS clinic from September 11, 2012 to September 10, 2016 were included.

\section{Exclusion Criteria}

All TB patients with incomplete data in the log book of the DOTS clinic and previously analyzed records, before September 11, 2012, were excluded.

\section{Data Collection and Processing}

Data were extracted by reviewing the profiles of all TB patients in the registry log book, using a data extraction sheet, at the DOTS clinic.

\section{Laboratory Methods}

The diagnosis of TB was performed by a conventional acid-fast bacilli (AFB) staining technique only in the first year, and by both an AFB technique and GeneXpert MTB/RIF assay for the remaining 3 years. 


\section{Acid-Fast Staining}

Mycobacterium contains mycolic acid in the outer membrane, making the cells waxy and resistant to staining with aqueous-based stains such as the Gram stain. But it stains by an alternative staining technique called acid-fast staining. The primary stain, carbol fuchsin, is applied to the cells, and heat and phenol are used to allow the stain to penetrate into the waxy surface of acid-fast microorganisms. The excess stain is removed with treatment by $3 \%$ acid alcohol. A secondary stain, methylene blue, is then applied to the cells. Under a $100 \times$ oil immersion objective, AFB are seen as pink to red in color.

\section{Xpert MTB/RIF Assay}

Sputum samples were collected and processed by the GeneXpert test, according to the manufacturer's instructions. The sample reagent was added in a $2: 1$ ratio $(1.5 \mathrm{~mL}$ of bactericidal sample reagent with $0.5 \mathrm{~mL}$ of specimen) to samples in a $15 \mathrm{~mL}$ falcon tube, and the tube was manually agitated twice and incubated for $15 \mathrm{~min}$ at room temperature. Then, $2 \mathrm{~mL}$ of the inactivated material was transported to a cartridge using a sterile disposable pipette. Cartridges were loaded into the Xpert assay device and results were interpreted as per the standard protocol. ${ }^{14,15}$

\section{HIV Testing}

A national HIV test algorithm was used for the diagnosis of HIV/AIDS infection to detect HIV antibody in a blood sample. At the time, the algorithm consisted of three HIV test kits, namely KHB, Stat pack, and Unigold, with different sensitivities and specificities.

\section{Data Analysis}

The collected data were checked for completeness, cleaned, coded, and analyzed using SPSS version 22. Logistic regression was used to determine the relationships between independent variables and the outcome variable. The odds ratio and $95 \%$ confidence interval were calculated and results were considered statistically significant at $p<0.05$.

\section{Ethical Approval}

The study was approved by the research and ethical review committee (RERC) of Debre Markos University (protocol number: DMU/CHSRERC/87/2017). Permission was secured from Debre Markos Comprehensive Specialized Hospital. Data were anonymized or maintained with confidentiality. The research and ethical review committee of
Debre Markos University waived the need for informed consent because the data were extracted retrospectively from the log book of the TB treatment center and confidentiality was maintained. No personal identification or labeling was used, and this study complied with the Declaration of Helsinki.

\section{Definitions of Terms}

- Smear-positive pulmonary tuberculosis (SPPTB): A patient with at least two sputum samples which were positive for AFB by microscopy, or a case with only one sputum sample which was positive for AFB by microscopy, and stable chest radiographic anomalies characteristic of active PTB. ${ }^{16}$

- Smear-negative pulmonary tuberculosis (SNPTB): A patient with signs indicative of $\mathrm{TB}$, with at least two sputum samples negative for AFB by microscopy, and with stable chest radiographic anomalies characteristic of active PTB, or a patient with at least two sputum samples taken at least 2 weeks apart. ${ }^{17}$

- Extrapulmonary tuberculosis (EPTB): TB of organs other than the lungs; for example, lymph nodes, abdomen, skin, joints and bones, and meninges. ${ }^{16}$

- TB/HIV co-infection: A patient diagnosed as having either PTB or EPTB concomitantly with a diagnosis of HIV/AIDS infection. ${ }^{17}$

\section{Results \\ Socio-Demographic Characteristics of Study Participants}

A total of 180 TB patients were enrolled and included in this study. Males constituted 96/180 (53.3\%) of the study participants. Altogether, 100/180 (55.6\%) of the study participants were in the age group 18-30 years, followed by 64/ $180(35.6 \%)$ in the age group $>30$ years and $16 / 180(8.9 \%)$ in the age group $<18$ years. The patients had a mean, standard deviation, and median age of 29.98, 12.82, and 27.00 years, respectively. Most of the patients, 133/180 (62.8\%), lived in urban areas. PTB accounted for 97/180 (53.9\%) of the cases. There were 164/180 (91.1\%) new incidence cases and $16 / 180(8.9 \%)$ treatment failures, but no relapsing or defaulting cases were observed (Table 1).

\section{Prevalence of HIV/TB Co-Infections}

The overall prevalence of HIV among TB patients was 72/ 180 (40\%) (Table 1). The proportions of HIV co-infections among TB patients were 41/72 (42.7\%) in males and 31/72 
Table I Socio-Demographic Characteristics of the Study Participants Registered at the DOTS Clinic in Debre Markos Comprehensive Specialized Hospital, Northwest Ethiopia, from September II, 2012 to September 10, 2016

\begin{tabular}{|l|l|l|l|}
\hline Variables & $\begin{array}{l}\text { Categorized } \\
\text { Variables }\end{array}$ & Frequency & $\begin{array}{l}\text { Percentage } \\
\text { (\%) }\end{array}$ \\
\hline Sex & Male & 96 & 53.3 \\
& Female & 84 & 46.7 \\
\hline Age & $<18$ years & 16 & 8.9 \\
& $18-30$ years & 100 & 55.6 \\
& $>30$ years & 64 & 35.6 \\
\hline Residence & Urban & 113 & 62.8 \\
& Rural & 67 & 37.2 \\
\hline TB type & PTB positive & 38 & 21.1 \\
& PTB negative & 59 & 32.8 \\
& EPTB & 83 & 46.1 \\
\hline TB & New & 164 & 91.1 \\
category & Relapsed & 0 & 0 \\
& Treatment failure & 16 & 8.9 \\
& Defaulter & 0 & 0 \\
\hline HIV status & HIV positive & 72 & 40 \\
& HIV negative & 108 & 60 \\
\hline
\end{tabular}

$(36.9 \%)$ in females; $31.2 \%$ in the $<18$ year age group and $56.2 \%$ in the $>30$ year age group; $40.7 \%$ in urban and $38.8 \%$ in rural residences; $50.0 \%$ in PTB smear positive, $47.5 \%$ in PTB smear negative, and $30.1 \%$ in EPTB; $42.1 \%$ in the category of new cases and $18.8 \%$ in the treatment failure TB patient category (Table 2). According to this study, the proportion of TB/HIV co-infection was 28/66 $(42.4 \%)$ in $2013,18 / 38(47.4 \%)$ in $2014,13 / 32$ (40.6\%) in 2015, and 13/44 (29.5\%) in 2016 (Figure 1).

\section{Associated Factors of TB/HIV Co-Infections}

The odds of having TB/HIV co-infection were 2.3 and 2.1 times higher in PTB smear-positive and PTB smear-negative patients, respectively, than in patients with EPTB in the logistic regression. In multivariate logistic regression analysis (controlling for the effect of confounding factors), PTB smear-positive and PTB smear-negative patients were significantly associated with HIV co-infection $(\mathrm{AOR}=3.2,95 \%$ CI 1.41-8.10, $p=0.006$; and $\mathrm{AOR}=2.1,95 \%$ CI 1.0-4.3, respectively, $p=0.05$ ) in comparison to EPTB patients; however, sex, age, residence, and TB category were not significantly associated with the co-infection (Table 2).

\section{Discussion}

The overall prevalence of HIV co-infection among TB patients was $40 \%$ (Table 2). This is comparable with the rates found in previous studies conducted in a similar area, at Debre Markos Comprehensive Specialized Hospital, at $44 \%,{ }^{18}$ in Nigeria, $44.2 \%,{ }^{19}$ and in Zimbabwe, $44 \%,{ }^{20}$ but lower than in studies conducted in Cameron, $51.6 \%,{ }^{21}$ and

Table 2 Binary and Multiple Logistic Regression Analysis of Socio-Demographic Characteristics and HIV Status of TB Patients at Debre Markos Comprehensive Specialized Hospital, Northwest Ethiopia, September II, 2012 to September 10, 2016

\begin{tabular}{|c|c|c|c|c|c|c|c|c|c|}
\hline \multicolumn{2}{|l|}{ Variables } & \multicolumn{2}{|c|}{ TB/HIV Co-Infection } & \multirow{4}{*}{$\begin{array}{l}\text { Total } \\
96 \\
84\end{array}$} & \multirow{4}{*}{$\begin{array}{l}\% \\
53.3 \% \\
46.7 \%\end{array}$} & \multirow{4}{*}{$\begin{array}{l}\text { COR }(95 \% \mathrm{CI}) \\
\mathrm{I} \\
0.78(0.43-1.43)\end{array}$} & \multirow{4}{*}{$\begin{array}{l}p \text {-Value } \\
0.428\end{array}$} & \multirow[t]{4}{*}{ AOR $(95 \% \mathrm{Cl})$} & \multirow[t]{4}{*}{$p$-Value } \\
\hline & & \multirow{3}{*}{$\begin{array}{l}\text { Yes } \\
41 \text { (42.7\%) } \\
31 \text { (36.9\%) }\end{array}$} & \multirow{3}{*}{$\begin{array}{l}\text { No } \\
55(57.3 \%) \\
53(63.1 \%)\end{array}$} & & & & & & \\
\hline Sex & Male & & & & & & & & \\
\hline & Female & & & & & & & & \\
\hline \multirow[t]{3}{*}{ Age } & $<18$ years & $5(31.2 \%)$ & II (68.8\%) & 16 & $8.9 \%$ & I & & & \\
\hline & $18-30$ years & 31 (3I.0\%) & $69(69.0 \%)$ & 100 & $55.6 \%$ & $00.98(0.3 \mathrm{I}-3.08)$ & 0.984 & & \\
\hline & $>30$ years & $36(56.2 \%)$ & $28(43.8 \%)$ & 64 & $35.6 \%$ & $2.82(0.88-9.08)$ & 0.081 & & \\
\hline \multirow[t]{2}{*}{ Residence } & Urban & $46(40.7 \%)$ & 67 (59.3\%) & 113 & $62.8 \%$ & I & & & \\
\hline & Rural & $26(38.8 \%)$ & 41 (6I.2\%) & 67 & $37.2 \%$ & $0.92(0.49-I .7 I)$ & 0.801 & & \\
\hline \multirow[t]{3}{*}{ TB type } & PTB +ve & $19(50.0 \%)$ & 19 (50.0\%) & 38 & $21.1 \%$ & $2.32(1.05-5.11)$ & $0.037^{*}$ & $3.2(1.4 I-8.10)$ & $0.006 *$ \\
\hline & PTB -ve & $28(47.5 \%)$ & 31 (52.5\%) & 59 & $32.8 \%$ & $2.09(1.04-4.19)$ & $0.039 *$ & $2.1(1.01-4.30)$ & $0.050 *$ \\
\hline & EPTB & $25(30.1 \%)$ & $58(69.9 \%)$ & 83 & $46.1 \%$ & I & & I & \\
\hline \multirow[t]{2}{*}{ TB category } & New & $69(42.1 \%)$ & 95 (57.9\%) & 164 & $91.1 \%$ & I & & & \\
\hline & Treatment failure & $3(18.8 \%)$ & $13(81.2 \%)$ & 16 & $8.9 \%$ & $0.32(0.08-1.15)$ & 0.082 & & \\
\hline
\end{tabular}

Notes: *Significant at $p<0.05$

Abbreviations: COR, crude odds ratio; AOR, adjusted odds ratio; PTB +ve, pulmonary tuberculosis smear positive; PTB -ve, pulmonary tuberculosis smear negative. 


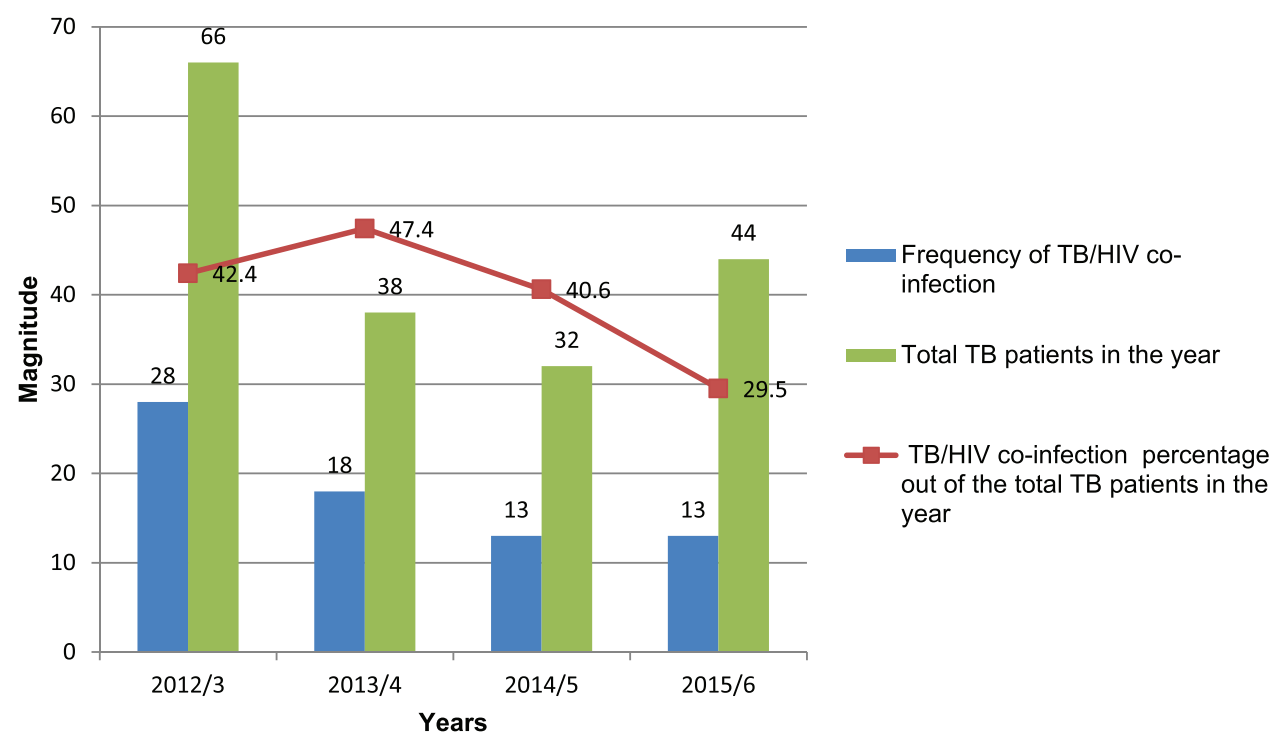

Figure I The pattern of TB/HIV co-infection at the directly observed treatment, short-course (DOTS) clinic, Debre Markos Comprehensive Specialized Hospital, Northwest Ethiopia, from September II, 2012 to September 10, 2016.

in Ghana, 46.2\%. ${ }^{22}$ However, this finding is higher than in other studies in Ethiopia, at Finote Selam Hospital, $22.1 \%,{ }^{23}$ Kobo-Robit and Gobiye, $24.3 \%,{ }^{24}$ and Dabat, $11.4 \%,{ }^{25}$ in the WHO report of $2015,12 \%,{ }^{2}$ and in Gamo Goffa, Ethiopia, $6.8 \%{ }^{26}$ This variation could be explained in terms of socio-demographic, time, or sample size variation, differences in individual disease burden, preference of patients for quality of care, hygiene practices of the population, and differences in socio-economic activity of the community.

HIV/TB co-infection has shown a declining trend in the past 2 years in the study area, in agreement with studies conducted at Finote Selam Hospital and Wellega. ${ }^{23,27}$ This may be due to increased awareness by HIV-infected patients about ART drugs and the prevention of opportunistic infections, so that their immunity may lift from having active TB from latent infection. Other reasons may be the expansion of health facilities across the country and the increased awareness of the community through health education, according to current strategic plan of the federal ministry of health of Ethiopia.

In this study, sex, age, residence, and TB category were not significantly associated with TB/HIV coinfection. Similar results were noted in studies conducted in Southern Ethiopia, ${ }^{26}$ Debre Markos Comprehensive Specialized Hospital, ${ }^{18}$ and Finote Selam Hospital, ${ }^{23}$ which found that sex, residence, and TB category were not significantly associated with TB/HIV co-infection. However, the odds of having TB/HIV co-infection were
3.2 and 2.1 times higher among PTB smear-positive and PTB smear-negative patients, respectively, compared with patients with EPTB (Table 2). That is, in multiple logistic regression analysis, being PTB smear-positive and PTB smear-negative was significantly associated with HIV infection in TB patients. This unusual association of PTB positive and negative smears with HIV coinfection could be due to the small number of EPTB patients admitted and participating in this study. Because of its subclinical features, EPTB patients often do not require admission. A similar result was obtained in a study at Kobo-Robit and Gobiye in Northern Ethiopia. $^{24}$ In addition, HIV suppresses the immunity of TB patients, resulting in latent TB, which creates a challenge in the diagnosis of TB through routine smearing techniques; this is why negative PTB smears also have an association with HIV co-infection.

In contrast to this finding, negative PTB smears were significantly more associated with HIV than positive PTB smears and EPTB patients in previous research findings from Debre Markos Comprehensive Specialized Hospital $^{18}$ and Dabat. ${ }^{25}$ In the case of the current study, this may be due to multiple factors; for example, if PTB smear-positive and/or HIV co-infected individuals adhered well to ART and cotrimoxazole preventive therapy (CPT), they would have a high level of immunity. ${ }^{4}$ This could be explained in terms of the role of host immunity in the pathogenesis of TB. Therefore, these individuals would have a high probability of being smear positive. 


\section{Limitations of the Study}

The data were collected using a secondary data source from already recorded documents, so there were some difficulties in obtaining all the information needed to characterize the risk factors. A prospective study design would be a better way to deal with this type of research interest.

\section{Conclusions}

This study indicated higher rates of TB/HIV co-infection than in other studies held in different parts of the country, even though the rate of TB/HIV co-infection in the study area has decreased from $44 \%$ to $40 \%$ in the past 4 years. TB/HIV co-infection was significantly associated with the type of TB. On the other hand, sex, age, residence, and TB category were not significantly associated with TB/HIV co-infection. Ethiopia's current collaborative TB/HIV prevention strategy is showing promise in the reduction of TB/HIV co-infection rates. Therefore, Ethiopia should strengthen the current collaborative TB/HIV prevention strategy to sustain this decrease in co-infection. The data recording system for patients should be improved to include patients' marital status, occupation, income, and personal lifestyle, such as addictions and co-morbidities. Researchers could also conduct a prospective study to obtain full information for further explanation of the higher proportions of TB/HIV coinfections compared to other studies.

\section{Abbreviations}

AFB, acid-fast bacilli; DOTS, directly observed treatment, short course; EPTB, extrapulmonary tuberculosis; HIV, human immunodeficiency virus; PTB, pulmonary tuberculosis; TB, tuberculosis.

\section{Data Sharing Statement}

All data generated and analyzed during this study were included in the manuscript.

\section{Ethical Approval}

The study was approved by the research and ethical review committee of Debre Markos University(protocol number: DMU/CHSRERC/87/2017). Data were maintained with confidentiality. There was no personal identification left on the check list.

\section{Acknowledgments}

We would like to acknowledge the TB/HIV treatment center staff of Debre Markos Comprehensive Specialized Hospital.

\section{Author Contributions}

All authors made a significant contribution to the work reported, whether that is in the conception, study design, execution, acquisition of data, analysis and interpretation, or in all these areas; took part in drafting, revising or critically reviewing the article; gave final approval of the version to be published. They have agreed on the journal to which the article has been submitted; and agree to be accountable for all aspects of the work.

\section{Disclosure}

All authors declare that they have no conflicts of interest in this work.

\section{References}

1. WHO. Global Tuberculosis Report 2019. Geneva: World Health Organization; 2019. Available from: https://www.who.int/teams/glo bal-tuberculosis-programme/tb-reports/global-report-2019. Accessed March 5, 2021.

2. World Health Organization. Global Tuberculosis Report 2015. 20th ed. World Health Organization; 2015. Available from: http://www. who.int/iris/handle/10665/191102. Accessed March 5, 2021.

3. CDC. Managing Drug Interactions in the Treatment of HIV-Related Tuberculosis; Centers for Disease Control and Prevention Office of Infectious Diseases National Center for HIV/AIDS, Viral Hepatitis, STD, and TB Prevention Division of Tuberculosis Elimination. 2013.

4. Kasper DL, Hauser SL, Jameson JL, Fauci AS, Longo DL, Loscalzo J. Harrison's Principles of Internal Medicine. 19th ed. New York: McGraw-Hill Education; 2016.

5. Jain SK, Aggarwal JK, Rajpal S, Baveja U. Prevalence of HIV infection among tuberculosis patients in Delhi: a Sentinel Surveillance Study. Ind J Tub. 2000;47(21):22-26.

6. EFMOH. Guidelines for Clinical and Programmatic Management of $T B, T B / H I V / A I D S$ and Leprosy in Ethiopia. 5th ed. Addis Ababa: Federal Ministry of Health; 2013.

7. FMOH. HIV Prevention and Control Office: Single Point HIV Prevalence Estimate. Addis Ababa, Ethiopia; 2007. Available from: http://www.etharc.org/aidsineth/publications/singlepointprev. Accessed March 5, 2021.

8. MacPherson P, Lebina L, Motsomi K, et al. Prevalence and risk factors for latent tuberculosis infection among household contacts of index cases in two South African provinces: analysis of baseline data from a cluster-randomised trial. PLoS One. 2020;15(3): e0230376. doi:10.1371/journal.pone. 0230376

9. WHO. Consolidated Guidelines on the Use of Antiretroviral Drugs for Treating and Preventing HIV/AIDS Infection: Recommendations for a Public Health Approach. Geneva: World Health Organization; 2013.

10. EFMOH. Ethiopian Federal Ministry of Health. Preliminary report of Ethiopia national TB/HIV sentinel surveillance. One year Report (July 2011 - June 2012). 2013.

11. Corbett EL, Watt CJ, Walker N, et al. The growing burden of tuberculosis: global trends and interactions with the HIV epidemic. Arch Intern Med. 2003;163(9):1009-1021. doi:10.1001/ archinte.163.9.1009

12. Herce ME, Muyoyeta M, Topp SM, Henostroza G, Reid SE. Coordinating the prevention, treatment, and care continuum for HIV-associated tuberculosis in prisons: a health systems strengthening approach. Curr Opin HIV AIDS. 2018;13(6):492-500. doi:10.1097/COH.0000000000000505 
13. CSA. The population and housing census of Ethiopia: statistical report for country level. Booklet Report; 2007.

14. WHO. Automated Real-Time Nucleic Acid Amplification Technology for Rapid and Simultaneous Detection of Tuberculosis and Rifampicin Resistance: Xpert MTB/RIF System. Policy Statement. Geneva: World Health Organization; 2011.

15. Zeka AN, Tasbakan S, Cavusoglu C. Evaluation of the GeneXpert MTB/RIF assay for rapid diagnosis of tuberculosis and detection of rifampin resistance in pulmonary and extrapulmonary specimens. J Clin Microbiol. 2011;49(12):4138-4141. doi:10.1128/JCM.0543411

16. WHO. Management of TB and HIV Co-Infection Clinical Protocol for the European Region. 4th ed. 2013. Available from: https://www. euro.who.int/_data/assets/pdf_file/0004/218515/. Accessed March 5, 2021.

17. EFMOH. Ethiopia National Treatment Guideline for TB in Pediatric. 2014.

18. Esmael A, Wubie M, Tsegaye G, Endris M. Tuberculosis and human immunodeficiency virus co-infection in debre markos referral hospital in Northwest Ethiopia. A Five Years Retrospective Study. J AIDS Clin Res. 2013;4:263. doi:10.4172/2155-6113.1000263

19. WHO. TB control and prevention guideline. 2010. Available from: https://apps.who.int/iris/bitstream/handle/10665/44425/ 9789241564069_eng.pdf?sequence=1. Accessed March 5, 2021.

20. Houston S, Ray S, Mahari M, et al. The association of tuberculosis and HIV infection in Harare, Zimbabwe. Tuber Lung Dis. 1994;75:220-226. doi:10.1016/0962-8479(94)90012-4
21. Pefura Yone EW, Kuaban C, Kengne AP. HIV testing, HIV status and outcomes of treatment for tuberculosis in a major diagnosis and treatment centre in Yaounde, Cameroon: a retrospective cohort study. BMC Infect Dis. 2012;12:190. doi:10.1186/1471-2334-12-190

22. Adjei AA, Adiku TK, Ayeh-Kumi PF, Hesse IF. Prevalence of human immunodeficiency virus infection among tuberculosis suspect patients in Accra, Ghana. West Afr J Med. 2006;25:38-41. doi:10.4314/wajm.v25i1.28243

23. Amare D. Tuberculosis and HIV Co-infection among patients on tuberculosis treatment at Fenote Selam District Hospital, Amhara Regional State, Northwest Ethiopia. Glob J Med Res. 2015;15 (5):2249-4618.

24. Mekonnen D, Deribe A, Desalegn E. TB/HIV co-infections and associated factors among patients on directly observed treatment short course in Northeastern Ethiopia. BMC Res Notes. 2015;8:666. doi:10.1186/s13104-015-1664-0

25. Tadesse S, Tadesse T. HIV co-infection among tuberculosis patients in Dabat, Northwest Ethiopia. J Infect Dis Immun. 2013;5(3):29-32. doi:10.5897/JIDI2013.0117

26. Zerdo Z. TB- HIV co-infection rate among smear positive pulmonary tuberculosis patients and associated risk factors in Southern Ethiopia. Sci Technol Arts Res J. 2014;3(3):87-92. doi:10.4314/star.v3i3.14

27. Ejeta E. HIV co-infection among tuberculosis patients on directly observed Treatment short course in Western Ethiopia. Nat Sci. 2014;12(9):68-72.
HIV/AIDS - Research and Palliative Care

\section{Publish your work in this journal}

HIV/AIDS - Research and Palliative Care is an international, peerreviewed open-access journal focusing on advances in research in HIV, its clinical progression and management options including antiviral treatment, palliative care and public healthcare policies to

\section{Dovepress}

control viral spread. The manuscript management system is completely online and includes a very quick and fair peer-review system, which is all easy to use. Visit http://www.dovepress.com/testimonials.php to read real quotes from published authors. 